more perplexing are the cases to which Erb's attention has lately been specially directed, in which specific spinal pachymeningitis has coincided with true tabetic degeneration. If the nature of the disease is such as I have suggested-if germs are deposited in the system which develop from time to time under influences of which we know nothing-it is easy to understand that the organismal processes, truly syphilitic, due to later germs, should coincide with degenerations which are the effect of toxins, the result of earlier broods.

Let me remind you, however, of one useful criterion of the existence of post-syphilitic degeneration which is often of service when you are puzzled by symptoms which may be due to either process. This is the isolated light-inaction of the iris-" isolated" because it still acts readily on accommodation. You are familiar with this, which is known by the name, as just as it is cumbersome, of the "Argyll-Robertson symptom." You know its association with tabes and general paralysis as degenerative sequelae of syphilis, but you may not know that it is met with in association with other conditions, and also as an isolated symptom. Even then it is of great significance as an indication of old syphilis, and I have many times had reason to appreciate the guidance it has afforded.

But these degenerative conditions are not within my present subject and I have reached the limit of time at my disposal. I have endeavoured to put before you some general principles which should be kept in mind. If you strive to apply them to cases that seem clear, you will find it easier to use them in cases that are obscure. It is necessary to accustom yourselves to analysing the symptoms in simple cases, and discerning the grounds on which the prognosis is founded and the treatment based, in order to obtain the ability to do so in cases that are complex. Even those that seem most difficult will generally yield to a deliberate effort to apply to them the principles I have given you ; they will yeild, if not altogether, at least in a degree sufficient for immediate need. Moreover you will. I believe, seldom fail to find that your conclusions are confirmed and your treatment justified by that to which we have all to look,

"The balance in the hand of time."

REFERENCE

\section{CASE OF ACUTE ACROMEGALY.}

BY W. MITCHELL STEVENS., M.D.LOND, M.R.C.P.LOND., Fellow of University College, London: Assistant Physician and Pathologist to the Cardiff Infirmary.

Since Pierre Marie in 1886 drew attention to the remarkable affection which he called acromegaly many cases of the disease have been recorded, until at the present time there exists an extensive literature on the subject. As acromegaly is a rare disease, the amount of writing on the subject of its pathology and clinical features may appear quite out of proportion to its real importance. Nevertheless, this disease is one of extreme interest, and its full investigation may tend to throw some light on the subject of "internal secretions," to which in late years the attention of many physicians has been directe $\mathrm{d}$.

The following case which was lately under my care is a typical one of what Sternberg calls "acute acromegaly," and in connexion with which he remarks: "In all cases with acute progress-and only in these cases-a true sarcoma of the hypophysis is present."

O. L. H. a married woman, aged $20-$ under the care of Dr. Roberts, of Pontypridd-came to my out-patient department at the Cardiff Infirmary on June 26th, 1902, complaining of severe headache, failure of sight, and alteration in personal appearance.

Family History.-Good.

Personal History.-Patient was a seven-months child, and her mother states that she was delicate until 12 months old, but after that time quite healthy until 14 years of age, when she commenced to suffer from occasional headaches of a mild character. Menstruation commenced when 14 years of age, and was quite regular till the onset of her illness. Patient was married two years ago, but has had no children.

History of Present Illness.-Three years ago (when 17 years of age) patient noticed that her eyesight commenced to fail and this was accompanied by very severe headaches. These symptoms becoming worse compelled her in two months time to give up her work in a post-office and they have gradually become more marked: The headaches though very severe, especially in the frontal region, have not been:constant, and she has frequently been free from them for days at a time, but the failure of eyesight has shown no remissions. She has never suffered from vomiting. Men- struation has entirely ceased since the commencement of the illness: For nearly twelve months before admission her friends had noticed that her face was becoming broader, her lips thicker and the nose enlarged and that her hands and feet were somewhat larger. During her illness she has been in good spirits and her mental powers have remained unimpaired except for occasional transitory loss of memory and confusion of ideas, On July ${ }_{3}$ rd she was admitted to the Cardiff Infirmary as an in patient under my care.

State on Admission.-She is a woman of average height, and although only 20 years of age, looks considerably more. Her type of face approaches that of the "negress." The skin of the face is coarse and thick and the nose large, broad and bulbous. The lips are thick and everted but the gums and teeth are normal. The tongue is broad and large, and its movements free. The hair on the head is thick, black and luxuriant The eyebrows are thick, black and well-defined. There is a marked de velopment of hair on the arms and legs and an increase of the norma amount in the axillae and on the pubes. The bones of the skull are not enlarged. The nasal bones and cartilages, especially the latter, are thickened. The lower jaw is thick and prominent, but the lower teeth do not project beyond the upper ones. The hands and feet are broad especially the phalanges. No other bones are affected. The thyroid is not apparently enlarged.

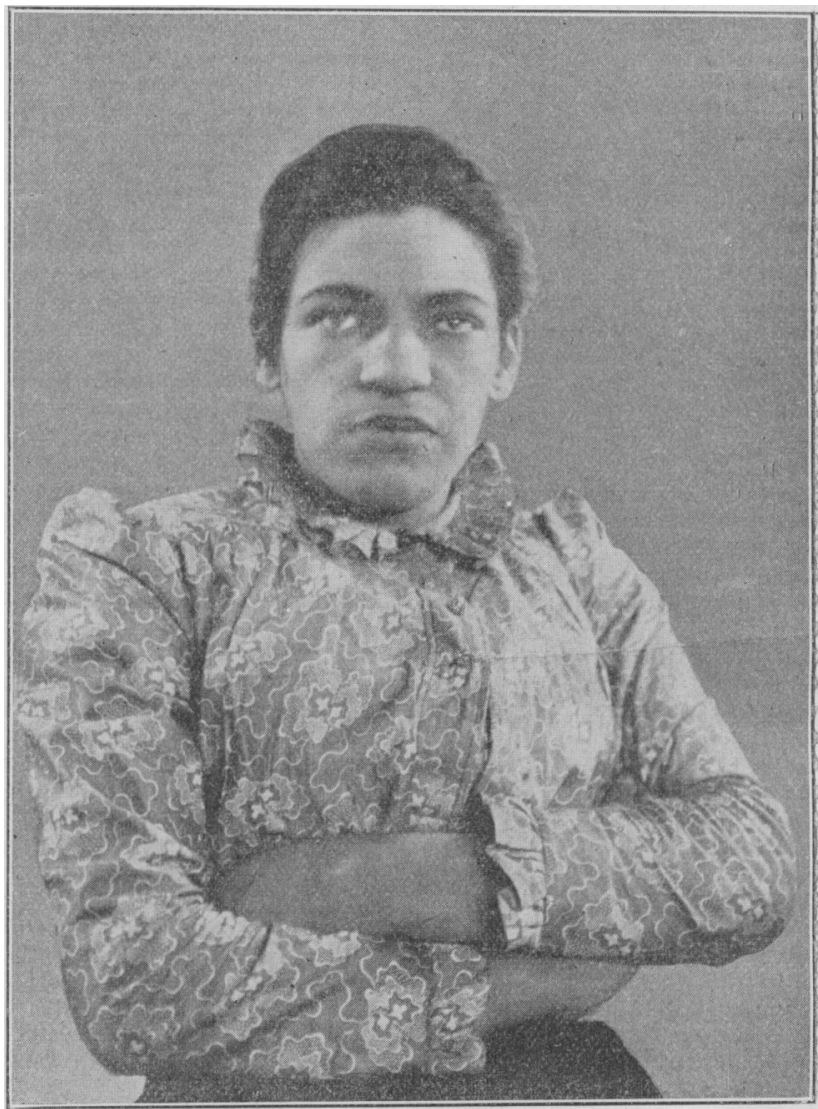

Fig. $x$.

Nervous System.-Memory is at times bad, and there is occasional con fusion of ideas. When free from headache she is bright and cheerful and quite intelligent. Speech is unaffected. Sleep is good, and patient is frequently rather drowsy. Headache is very severe, and though diffused over the whole head is most marked over the frontal region, and par ticularly severe above the left eyebrow, where it is associated with marked local tenderness. The headache is not constant, there being in tervals of freedom for some hours at a time. The reflexes are normal Muscular power is good. The special senses are all normal except the eyesight, which is very defective, the acuity of vision being, with right eye $\frac{1}{8}$, and with the left eye, $\frac{1}{3} \mathrm{~s}$. There is slight ptosis of the left lid, but the ocular movements are otherwise normal. There is well-marked bitemporal hemianopsia, and optic atrophy of both dises is present. The other organs are normal.

Progres8.-The patient after admission rapidly became worse. The headaches iccreased in severity, and though at first slightly relieved by phenacetin and other drugs, and by the administration of thyroid extract these had ro effect afterwards. The eyesight rapidly became worse untis the patient wasfpractically blind. She suffered also from occasiona vomiting, which was always associated with the taking of food or medi cine. Her features during the short time (four weeks) that she was under observation became much courser, and the increased enlargement of the lips was especially noted.

On July 24th (three weeks after admission) she had a severe "convulsion, 
with tonic spasm of roth sides of the body lasting for two minutes and followed by a semico natose state of several hours' duration. On account of the fearful severity of the headache, which resisted all medicinal remedies I asked my friend and colleague, Mr. Lynn Thomas, C.B., to trephine the skull with the object of relieving intracranial pressure.

On July 25 th he removed a piece of bone over the upper part of the left Rolandic area, and on its removal the brain, covered by dura mater, bulged considerably. The piece of bone removed was quite normal. The patient recovered from the operation, but died in a comatose condition on July zoth.

Necropsy.-I obtained permission to examine the brain only. A large tumour was found in the middle fossa at the base of the skull, occupying almost the whole of the space between the pons behind the frontal lobes in front and the temporo-sphenoidal lobes laterally. It measured 2 in. in length and $1 \frac{3}{4}$ in. in breadth. It was separated into right and left parts by a longitudinal depression. The left part, the larger and more pro

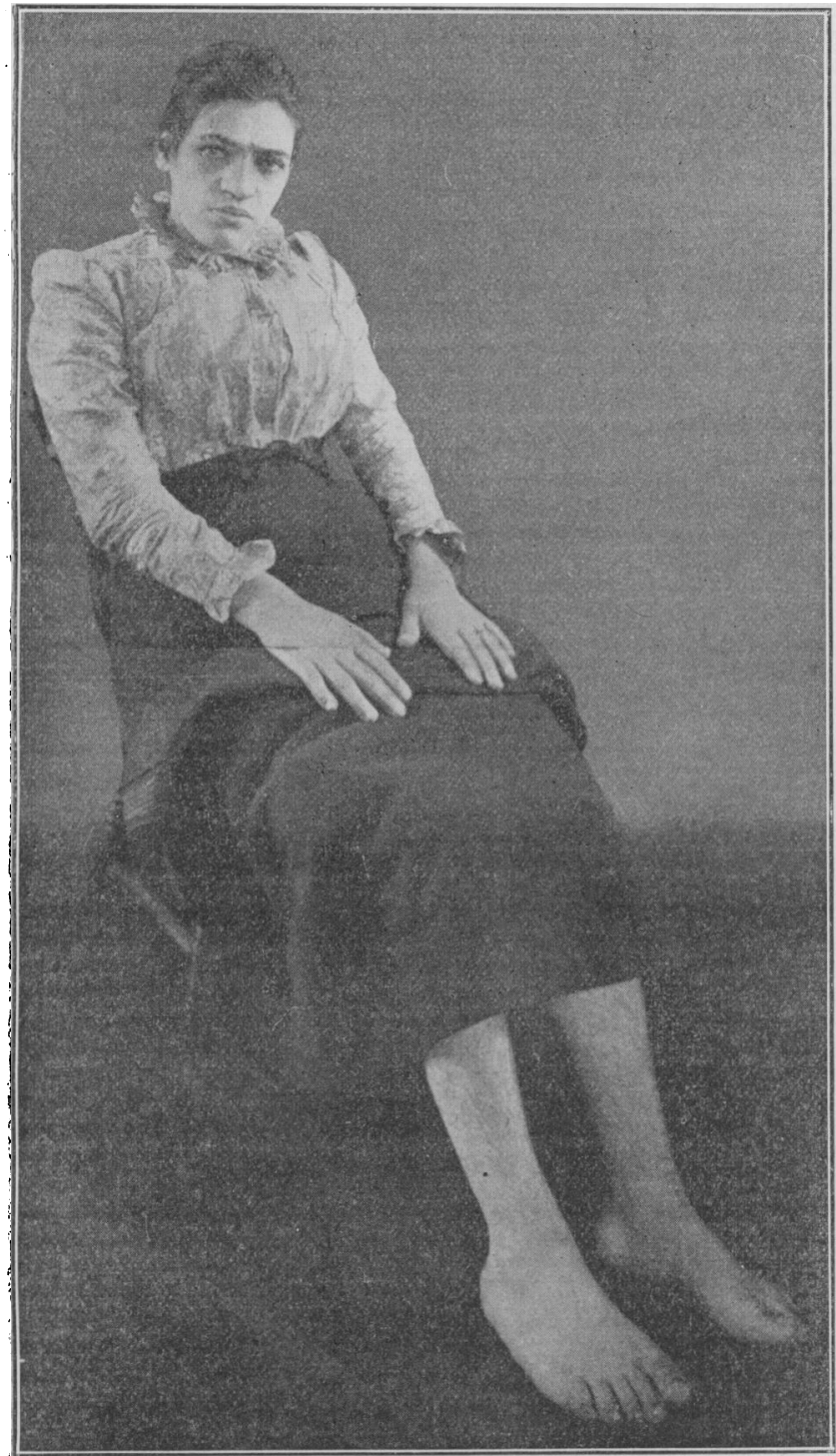

Fig. 2.

jecting one, was oval in shape, and reached inferiorly to theslevel of the tip of the temporo-sphenoidal lobe, which was somewhat compressed: the right part was rounder, smaller, and less projecting. The left part of the tumour was grey in colour, 'resembling ordinary brain substance except that it was firmer, and from its anterior part projected a red cyst the size of a hazel nut, which had a thin membranous wall, and contained soft and partially decolorized blood clot and a little yellow serous fluid. 'I'he right part was pale red in colour and cystic in parts.

On section the tumour was seen for the most part to be formed of grey homogeneous material, fairly firm on the surface but softer in the deeper parts, and on the right side were found several "blood cysts." From beneath the posterior border of the tumour there were several small projecting soft flaccid cysts like "squeezed and empty grapes," and these were found to be connected with the main tumour on the right side.

The tumour occupied the sella turcica and extended considerably begond $i$ t, and no trace of 'the pituitary body remained. The pituitary fossa was deeply eroded on bothisides," and the posterior cliroid processes had disappeared.

The olfactory nerves were unaffected. The optic chiasma was displaced towards the right side, and was found to be squeezed and flatlened out batween the projecting anterior end of the right part of the tumour and the right frontal lobe of the brain and to be greatly atrophied. Both optic nerves were thin and flattened and marked]y atrophied. The optic tracts were pressed on and more especially the left and were very small. Other cranial nerves were free from pressure though the left oculo-motor nerve was stretched,

Microscopical Examination.-The greater part of the tumour showed the appearance of a small round-celled sarcoma, the remaining parts being "haematomatous." The cranial bones, except for the effects of the pressure of the tumour, presented no"abnormal appearances.

This case was a typical one of acute acromegaly. It presented two classes of aymptoms :

I. Those due to a presence of an intracranial tumour in the position of the pituitary body, such as headache and visual defects.

2. Associated symptoms of acromegaly, namely, alterations in the face and enlargement of the hands and feet.

The progress of the disease during the last three weeks of life was very rapid, and it was interesting to observe that the facial appearances obviously altered within that space of time. Treatment had no effect, and my only hope was not to be able to arrest the disease, but, if possible, to relieve the symptoms

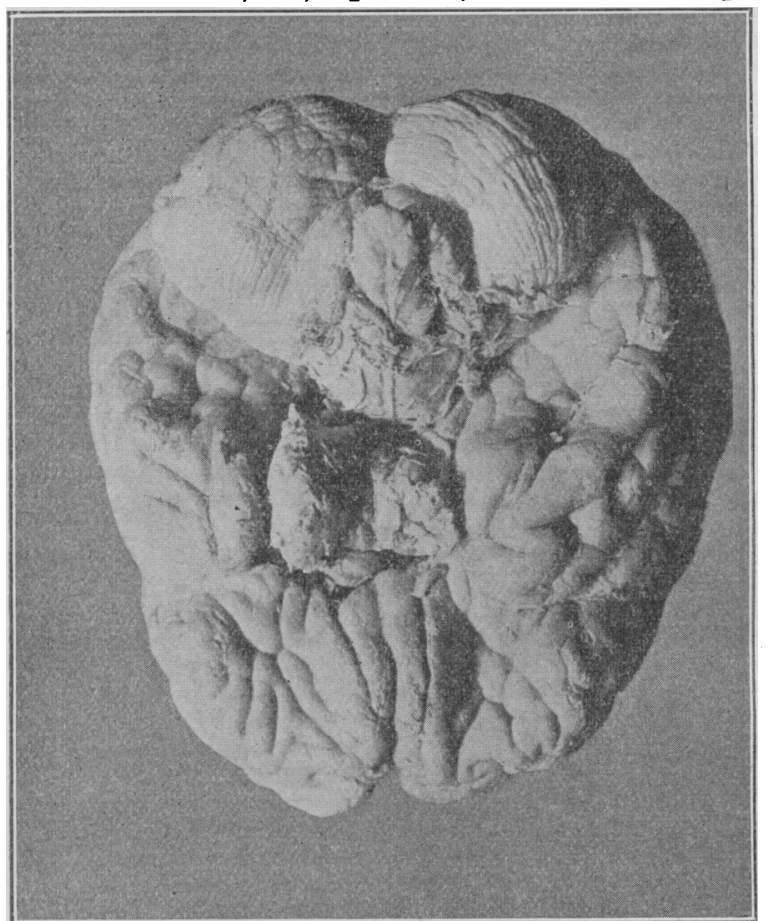

Fig. 3.-Photograph of brain showing tumour of the pituitary body. of intracranial pressure. In this connexion I may state that in the British Mredical Journal, 1896, vol. ii, p. 909, Mr. Lynn Thomas, C.B. reported a case of acromegaly, where the symptoms were greatly relieved by trephining.

In the acute cases of acromegaly, that is, those lasting from three to four years, in these only and in these always, there has been found a sarcoma of the pituitary body, and to this type belongs the case above described. Such acute cases must of necessity be fatal, as there is a malignant intracranial tumour, and the only hope is, if possible, to relieve the symptoms of intracranial pressure by "trephining."

Two classes of symptoms are present in cases of acromegaly as above mentioned-namely, those of an intracranial tumour and those properly termed "acromegalic." The first class are directly due to the enlargement of the pituitary body, and depend upon the amount of pressure exercised by the growth, and the second class of symptoms are peculiar and striking, are present to a greater or less extent, and are probably due to loss of function of the pituitary body.

The pathology of acromegaly has been much discussed, and has given rise to great divergence of opinion. The chief points are :

I. What is the cause of the acromegalic condition?

2. What are its relations to giantism ? 
These questions have been many times discussed, and I shall limit myself to pointing out some of the most salient points. Now in all cases of acromegaly there is disease of the pituitary body. This fact proves, of course, a direct relationship between the two conditions. The question then becomes: Is the acromegalic condition secondary to and dependent on disease of the pituitary body, or is the enlargement of this body (the diseased process always present) one of the phenomena of acromegaly? Some hold the latter view, and look upon the enlargement of the pituitary body as a partial phenomenon of acromegaly as, in fact, one of the symptoms of the disease analagous to the enlargement of the hands and feet. This view, however, seems to me an untenable one, for it certainly does not explain how a new growth like a sarcoma can give rise to acromegaly, and, moreover, in a large number of cases of acromegaly there is no "real " enlargement of the pituitary body, but rather an apparent one causing destruction of the gland itself, such as cystic tumours, colloid degenerations, etc. The balance of evidence certainly points to the conclusion that disease of the pituitary body with consequent loss of its function is the cause of acromegaly, and the development of the anterior lobe of this body from the oral diverticulum and its glandular structure suggest that it, like the thyroid gland. has some " secretory" function influencing the metabolism of the body. The main objections to this view are:(I) That tumours of the pituitary body may exist without symptoms of acromegaly; (2) that the medicinal use of pituitary extracts has little or no influence upon the course of the disease. It is quite true that tumours of the pituitary gland, and indeed tumours which may fairly be supposed to entirely destroy the functions of that body, may be present without causing symptoms of acromegaly. The very same objection has been raised against Addison's disease being dependent on an affection of the suprarenal capsules, for the latter may be so diseased as to be functionless, and this may occur without any symptoms pointing to Addison's disease. It is to be remembered, however, that acromegaly, like Addison's disease, is essentially "chronic" in its course, and death may occur before the development of definite symptoms apart from those of intracranial pressure. In the case above recorded it is certain that disease of the pituitary body had existed for three years and possibly longer, but definite symptoms of acromegaly (apart from those due to an intracranial tumour) were not observed tintil two years later, and had this patient died one year earlier the case would have been described as disease of the pituitary body. without acromegaly.

In the treatment of acromegaly there are two objects: (1) The treatment of the local condition, the pituitary tumour, upon the presence of which depends the symptoms of intracranial pressure, and the object is to relieve such pressure by diminishing the size of the fumour, preventing its grôth; or possibly removing it, and, lastly, the palliative one of "trephining"; (2) the treatment of the symptoms due to loss of function of the pituitary body-that is, acromegaly. In the treatment of this disease we cannot expect to obtain such good results as in the treatment of myxoedema, for in the latter there are no local symptoms due to the presence of a tumour. If we suppose that symptoms of acromegaly are due to loss of some secretion of the pituitary body, all that can be expected at the most from a pituitary extract is to influence those symptoms and to prevent further effects, and it can scarcely be expected to influence the pituitary tumour itself. Though good results have been described from the administration of pituitary extracts and from their combination with thyroid extracts, nevertheless their 'use has generally been most disappointing. In cases where the thyroid extract has proved beneficial it is probable that there has been some accompanying myxoedematous condition.

The want of success in the use of pituitary extracts in cases of acromegaly does not prove that this -disease is not due to loss of some "secretion" of the pituitary gland necessary to the economy. Even in the case of the thyroid, we must remember that, though the thyroid extracts are so beneficial in myxoedema in man, they do not prevent symptoms of that disease' in a monkey after thyroidectomy, though thyroid grafts are beneficial ; moreover, it is to be borne in mind that the active principle of the pituitary gland may be destroyed in the manufacture of the pituitary extracts. Even if it were possible to cure, relieve, or prevent the further progress of symptoms due to the loss of function of the pituitary gland, the problem of dealing with the intracranial tumour would still remain.

If this tumour is malignant, prospects are hopeless; and if simple, some reduction in its size might occur; and, indeed, in some recorded cases it is stated that under treatment the eyesight has recovered and the headache lessened.

The second question is, What are the relations of acromegaly to giantism? It is an interesting fact that about 20 per cent. of acromegalians are giants, and also that about i4 per cent. of cases of acromegaly commence under 20 years of age. It is also important to observe that when there is congenital absence of the thyroid, cretinism with dwarfing is present. These facts suggest that loss of pituitary function leads to overgrowth with late union of the epiphyses, and that loss of thyroid function has the opposite effect.

Although only a certain proportion of giants are acromegalians, it seems probable that all cases of acromegaly commencing in youth lead to giantism. Nevertheless, there are cases of acromegaly which begin in youth, and which show no giant growth, and this at first sight seems difficult to harmonize with the above suggestion; nevertheless, when it is considered how frequently disease of the pituitary and thyroid glands coexist, it is quite possible that the absence of giant growth in some cases of acromegaly in early life may be explained by a coexisting loss of function of the thyroid gland which may inhibit excesive growth. In any case, it cannot be denied that giantism is a not infrequent symptom of acromegaly.

\section{CASE OE CHRONIC ACROMEGALY.}

By C. H. CATTLE, M.D., M.R.C.P.,

Assistant Physician to the Nottingham General Hospital.

THE following notes, with the accompanying photographs, will serve to give a tolerably accurate description of the disease acromegaly, and although over 200 well-authenticated cases have already been placed on record, it must still bi regarded as a rare disease.

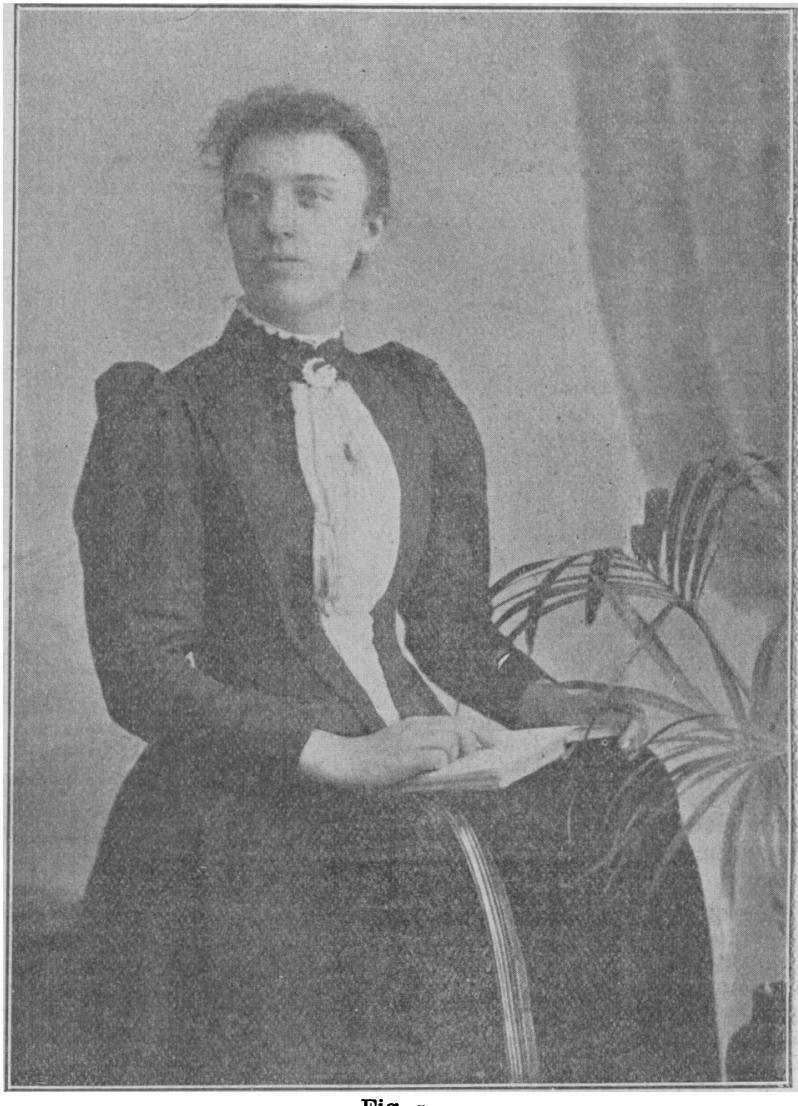

Fig. $x$.

History.-A. F., an unmarried woman, aged 30 , was first seen at the Nottingham General Hospital on July $x_{4}$ th, 1902. She stated that her health began to fail four years before, and that three years previously she had been treated at this hospital. The out patient notes of that date state, among other things, "face somewhat acromegalic." There was nothing of importance etiologically in the patient's personal or family history Since the present illness began the following have been the most 